\title{
LIGHT BACKSCATTERING BY ICE CRYSTALS OF CIRRUS CLOUDS WITHIN THE PHYSICAL OPTICS APPROXIMATION
}

\author{
Alexander Konoshonkin*, ${ }^{*}$, Anatoli Borovoi ${ }^{1}$, Natalia Kustova ${ }^{1}$, Zhenzhu Wang ${ }^{2}$, Dong Liu ${ }^{2}$, \\ Chenbo Xie ${ }^{2}$ \\ ${ }^{1}$ V.E. Zuev Institute of Atmospheric Optics SB RAS, Tomsk 634055, Russia \\ ${ }^{2}$ Key Laboratory of Atmospheric Optics, Anhui Institute of Optics and Fine Mechanics, Chinese Academy \\ of Sciences, Hefei 230031, China \\ *Email: sasha_tvo@iao.ru
}

\begin{abstract}
Lidar technologies are widely used for retrieving microphysics of cirrus clouds, i.e. sizes, shapes and spatial orientation of ice crystals constituting the clouds. Interpretation of the lidar signals are based on the backscattered light. However, properties of the light backscattered by the ice crystals have not been well known and understood yet. The reason of this is that the problem of light scattering by the ice crystals much larger that incident wavelengths has not been solved satisfactorily yet because of great demands to computer resources. In this contribution we review the physical optics approximation as a prospective method to solve the light scattering problem on large nonspherical particles in lidar application.
\end{abstract}

\section{INTRODUCTION}

There are several recently developed numerical exact methods based on the Maxwell equations like the PSDT (pseudo-spectral time domain method) and II-TM (invariant imbedding T-matrix method) [1,2]. At present, these methods are capable to solve the problem numerically for rather small ice crystals while the larger crystals can only be calculated within the physical-optics approximation [3]. The scattering problem is characterized by the size parameter $x=\pi a / \lambda$, where $a$ is the characteristic particle dimension and $\lambda$ is the incident wavelength. The maximum size parameter achieved within the numerical exact methods is reported as large as 100 in [1] and 130 in [2] while in natural cirrus clouds this parameter runs up to several thousands.

In this contribution, a review of the backscattered data obtained by the physical-optics approximation is proposed. One of the discussed topics is existence of a sharp peak around the backward scattering direction in the phase function of cirrus clouds. Earlier, such a peak was predicted within the physical-optics approximation [3]. It was explained as the cornerreflection effect caused by dihedral angles of 90 degrees between some crystal facets. Later this peak was obtained for smaller crystals as well by means of the numerical exact methods [1,2]. Moreover, the peak took place for the roughed crystals and irregular crystal shapes. Nevertheless, this problem has not completely clarified yet. In this paper, the new data obtained within the physical-optics approximation are presented that clarify whether the backscattering peak appears in cirrus clouds.

Lidar community usually does not operate with the phase function. The microphysics is inferred from the backscatter ratios: the depolarization, color, and lidar (extinction-to-backscatter) ratios. These quantities are main sources of information about size, shape and spatial orientation of the crystals [4]. In this contribution, these ratios are reviewed in application to the scanning and tilted lidars.

\section{CORNER REFLECTION EFFECT}

The corner reflection effect predominates in backscattering only for pristine hexagonal ice crystals. In real cirrus clouds the crystals often have a complicated shape, such as hollow columns, rough crystals and aggregates. These types of particles are often randomly oriented. This is the reason why lidars cannot observe the corner reflection effect in their everyday observations.

It is worthwhile noting that pristine hexagonal ice plates are frequently observed with their quasihorizontal special orientation [5-8]. In this case the vertically pointing lidar cannot detect the corner reflection effect because the backscatter mainly consists of the specular reflection. Only recently Veselovskii et al. [9] using the tilted lidar 
have observed the corner reflections effect of the quasi-horizontally oriented pristine ice plates. The observation showed that the backscattering Angström exponent (BAE) changed from zero to 0.55 and two depolarization ratios at two wavelengths diverged.

The information about the fraction of the quasihorizontally oriented pristine ice plates is important for radiative transfer models. Cirrus clouds consisted of either quasi-horizontally or randomly oriented particles with the same optical thickness produce essentially different radiative forcing.

The physical optics method allowing us to estimate the fraction of the quasi-horizontally oriented pristine ice plates by using the data from tilted lidar sensing linear depolarization ratio at two wavelengths. As an example, Fig. 1 shows the result of retrieving the fraction of quasihorizontally oriented pristine ice plates from data presented by Veselovskii et al. [9]. The retrieving can be made separately by using only the BAE (red line) or by using only two depolarization ratios (green and blue lines). The results agree well [10].

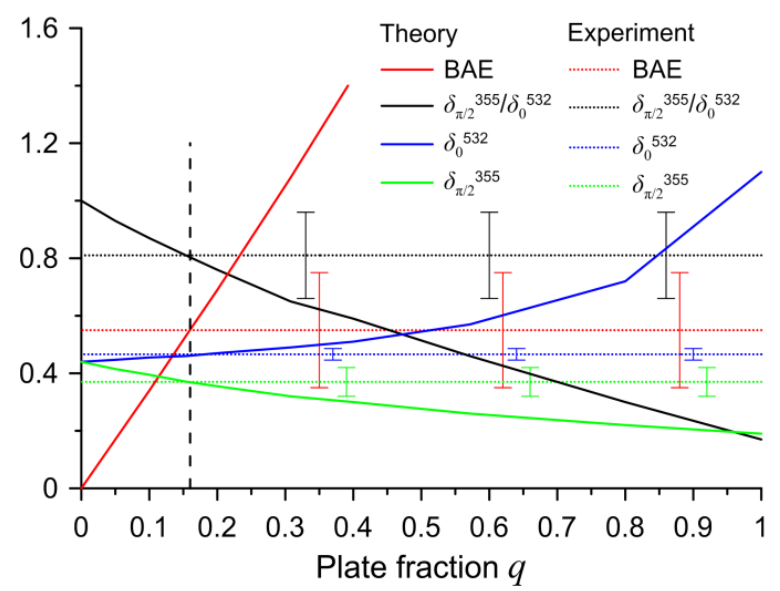

Figure 1. Experimental and theoretical data for the depolarization ratios and the backscattering Angström exponent (BAE) at the height of $8 \mathrm{~km}$.

\section{THE DATA BANK OF THE MUELLER MATRICES}

Within the physical optics method we have found the solution of the light scattering problem by ice crystal particles of cirrus clouds for particles with size varied from 10 to $1000 \mu \mathrm{m}$ at wavelengths from 0.355 to $1.6 \mu \mathrm{m}$.
The solution of the light scattering by randomly oriented hexagonal ice plates and columns in the backscattering direction is presented as a data bank of Mueller matrices. As an example, Fig. 2 shows the lidar and depolarization ratios calculated with help of this data bank for the hexagonal plates.
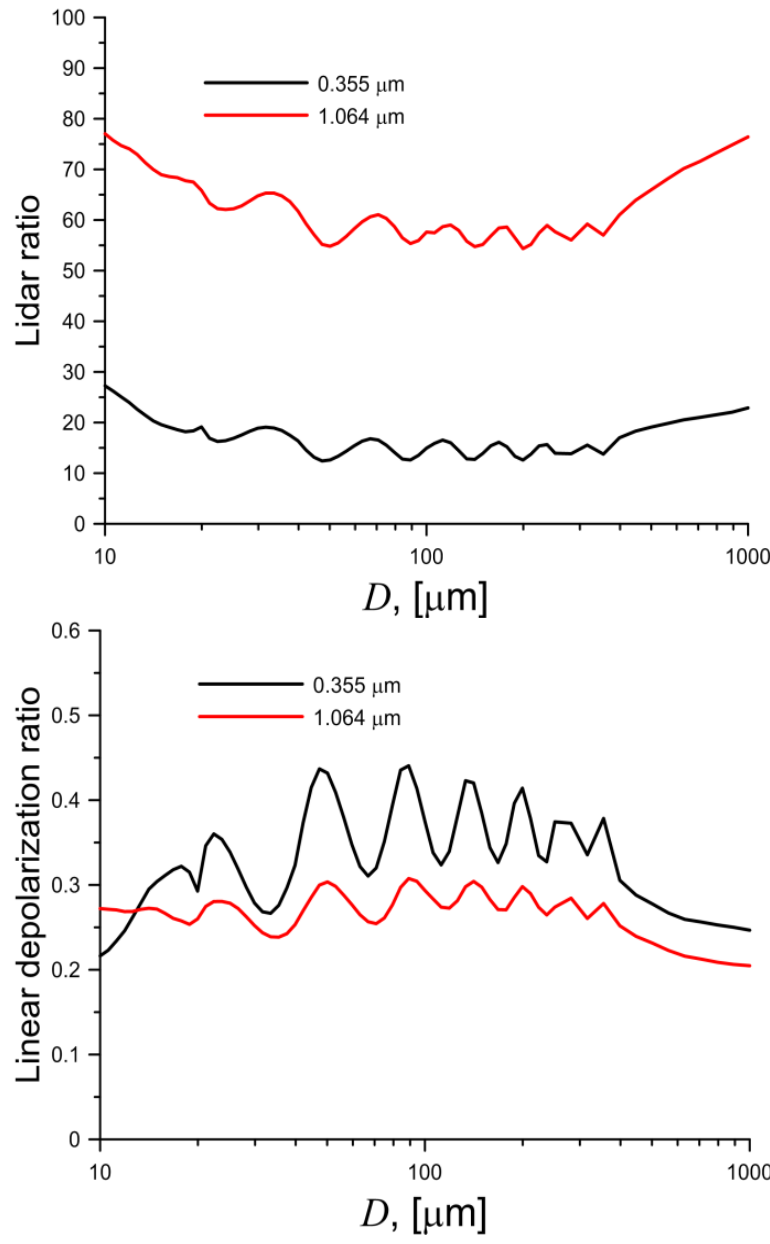

Figure 2. The lidar ratio and linear depolarization ratio for randomly oriented hexagonal ice plates as a function of the diameter (D) of the hexagonal facet for two wavelengths of 0.355 and 1.064 microns.

Figure 2 shows that the lidar ratio has a strong dependence on the wavelength of the incident light but it has a weak dependence on the particle size. The linear depolarization ratios, on the contrary, have a weak dependence on the wavelength but it significantly oscillates with particle size.

It is of great interest to study the dependence of the optical characteristics of an ensemble of ice particles as a function of the effective slope angle 
of particles relative to the horizontal plane, since the particles in clouds often have predominant orientation. The optical characteristics of predominantly oriented crystal particles obtained by us using the physical optics approximation are also presented in the data bank of the Mueller matrices. As an illustration, Fig. 3 shows the lidar and depolarization ratios for hexagonal columns and plates, as well as for the droxtals at a wavelength of $0.355 \mu \mathrm{m}$, depending on the effective slope angle $\beta_{\text {eff }}$ of the particle.
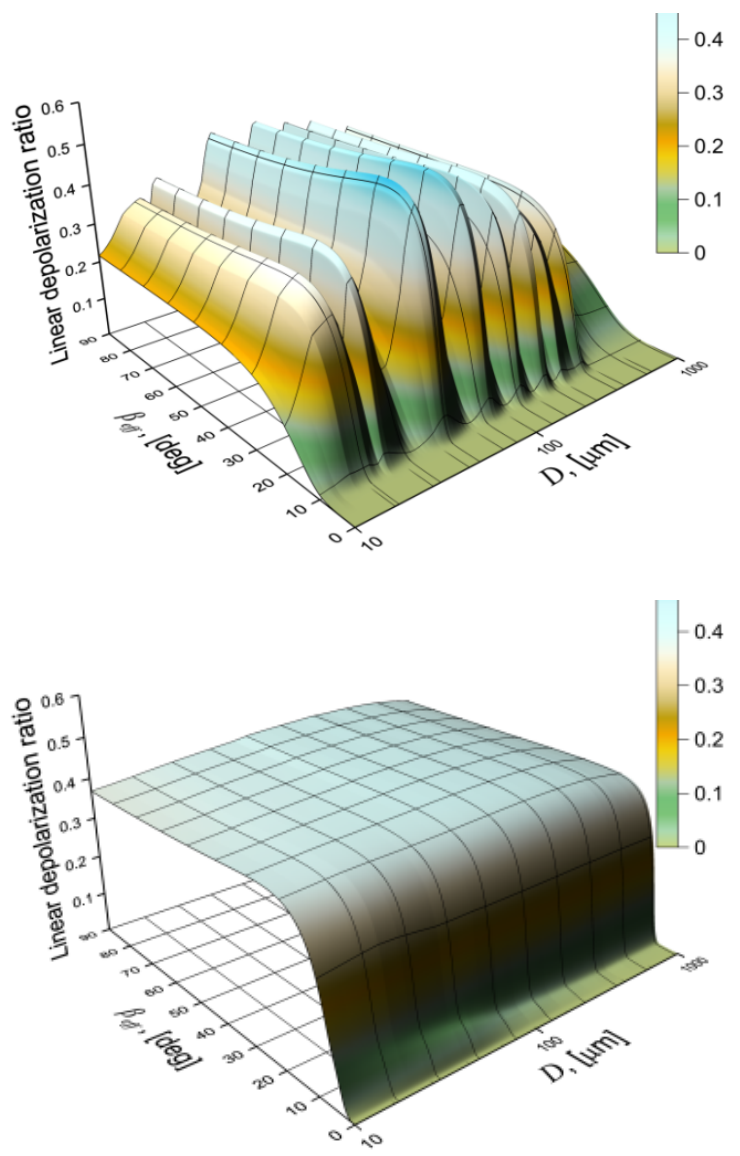

Figure 3. The linear depolarization ratio for quasihorizontally oriented hexagonal ice plates and droxtals.

\section{SCANNING LIDAR}

Based on the data bank we have already studied the signal of the scanning lidar. The example of this important result is shown in Fig. 4. Here we can see that there is the sharp peak of the lidar ratio and of the first element of the Mueller matrix $\left(M_{11}\right)$ in the vicinity of small lidar angles. It is important to analyze this vicinity for interpreting the scanning lidar data.
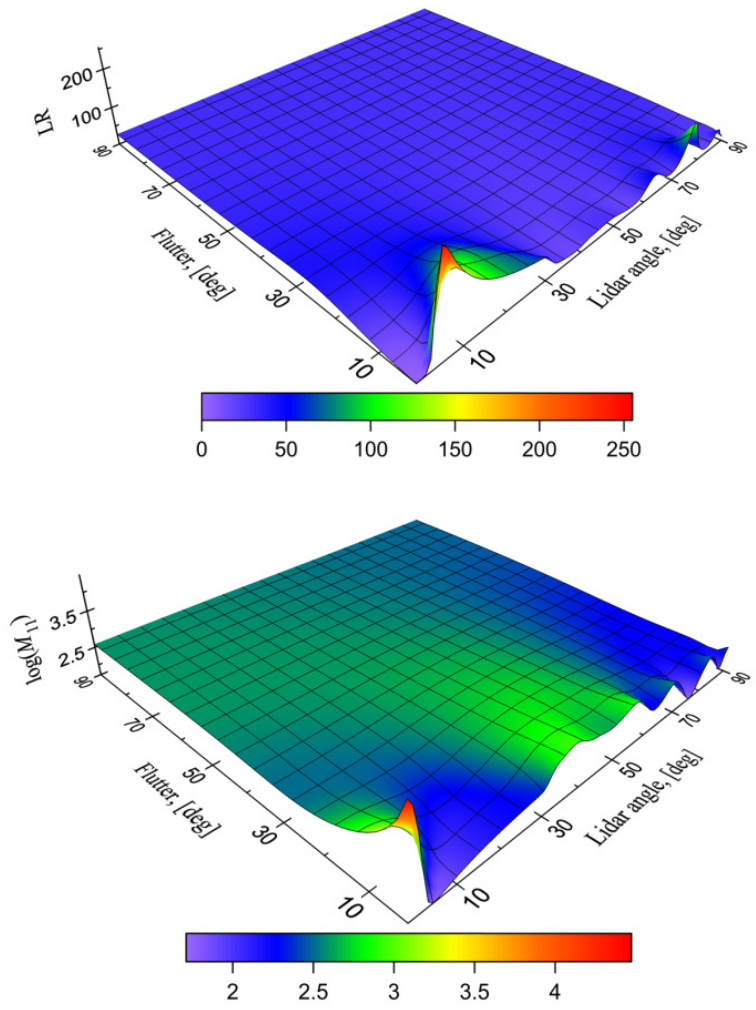

Figure 4. The lidar ratio (LR) and first element of the Mueller matrix for the cloud of ice plates with size of $100 \mu \mathrm{m}$ depending on the flutter and the lidar scanning angle.

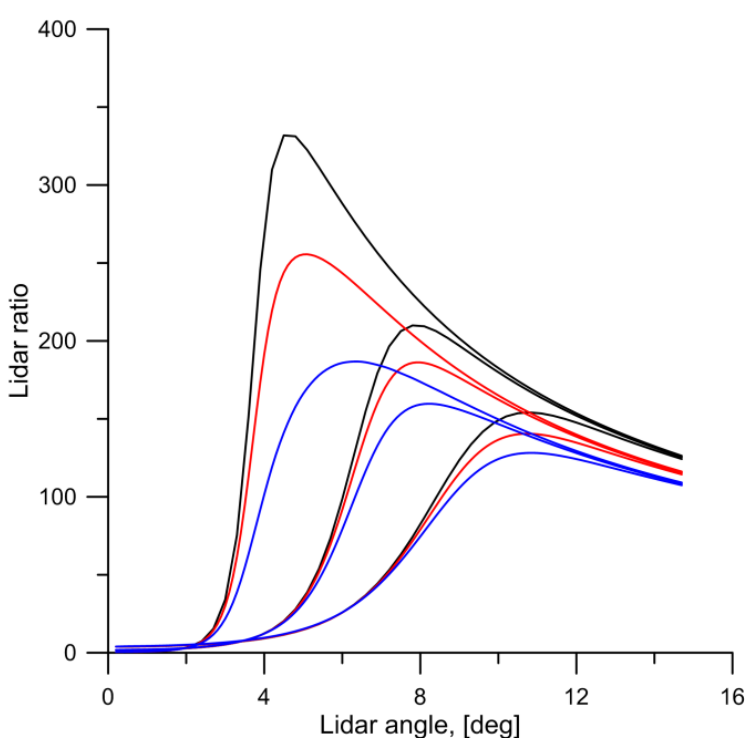

Figure 5. Comparison of the lidar ratio depending on the lidar angle for the plate with different size: $30 \mu \mathrm{m}$ (blue), $100 \mu \mathrm{m}$ (red) and $300 \mu \mathrm{m}$ (black). The flutter varies from 1 to 3 degrees (from left to right slopes). 
We have calculated the lidar ration for different sizes of particle and different effective tilt of the quasi-horizontally oriented pristine ice plates. The results are shown in Fig. 5. It is clearly seen that the size of the particle influence less than the particle orientation. Changing the size in 10 times (from 30 to 300 micron) leave the localization of the slope in the same place, while changing the flutter from $1^{\circ}$ to $2^{\circ}$ shifts the slope from 3 to 5 degrees of the lidar angle.

\section{CONCLUSION}

In this study we showed that the physical optics approximation can be successfully used to calculated the data bank of solutions of light scattering problem to interpret the lidar signals. Also scanning lidars are prospective tools to detecting the main parameter of horizontally oriented particles: the flutter, i.e. the degree of particle orientation. It is very important parameter that has to be added to the numerical models of the planet's radiation balance. It has been shown that the degree of particle orientation can be found directly from the lidar ratio profile of the scanning lidar. In this profile the lidar ratio has a strong slope and the localization of this slope gives the information of the flutter. It is also shown that the wavelength has little effect on the localization of the slope. The size of the particle, as far as the mixture of the randomly oriented particles, influence only to the size of the slope but not on the localization, so gives no difficulties to interpretation algorithms.

\section{ACKNOWLEDGEMENTS}

This work is supported by the RFBR under Grants no. 18-05-00568, no. 18-55-53046. Calculation of Mueller matrixes within the physical optics method is supported only by Russian Science Foundation (18-77-10035).

\section{REFERENCES}

[1] C. Zhou, P. Yang, "Backscattering peak of ice cloud particles", Opt. Exp. 23(9), 11995, 2015.

[2] J. Ding, P. Yang, et al., "Ice cloud backscatter study and comparison with CALIPSO and MODIS satellite data", Opt. Exp. 26(1), 620, 2016.

[3] A. Borovoi, A. Konoshonkin, N. Kustova, "Backscattering by hexagonal ice crystals of cirrus clouds," Opt. Lett. 38(15), 2881(2013).

[4] A. Konoshonkin, A. Borovoi, N. Kustova, J. Reichardts, "Power laws for backscattering by ice crystals of cirrus clouds," Opt. Exp. 25(19), 22341, 2017.

[5] J. Gasteiger, M. Wiegner, S. Groß, V. Freudenthaler, C. Toledano, M. Tesche, K. Kandler, "Modelling lidar-relevant optical properties of complex mineral dust aerosols", Tellus B: Chemical and Physical Meteorology 63(4), 725-741, 2011

[6] M. A. Yurkin, V. P. Maltsev, A. G. Hoekstra "The discrete dipole approximation for simulation of light scattering by particles much larger than the wavelength", J. Quant. Spectrosc. Radiat. Transfer 106, 546-557 (2007)

[7] A. Borovoi, A. Konoshonkin, N. Kustova "The physical-optics approximation and its application to light backscattering by hexagonal ice crystals", J. Quant. Spectrosc. Radiat. Transfer 146, 181-189 (2014)

[8] A. V. Konoshonkin, A. G. Borovoi, N. V. Kustova, H. Okamoto, H. Ishimoto, Y. Grynko, J. Förstner "Light scattering by ice crystals of cirrus clouds: From exact numerical methods to physical-optics approximation", J. Quant. Spectrosc. Radiat. Transfer $195,132-140(2017)$

[9] A. V. Konoshonkin, N. V. Kustova, A. G. Borovoi "Beam-splitting code for light scattering by ice crystal particles within geometric-optics approximation", J. Quant. Spectrosc. Radiat. Transfer 164, 175-183 (2015)

[10] A. G. Borovoi, A.V. Konoshonkin, N.V. Kustova, I.A.Veselovskii "Contribution of corner reflections from oriented ice crystals to backscattering and depolarization characteristics for off-zenith lidar profiling”, J. Quant. Spectrosc. Radiat. Transfer 212, 88-96 (2018) 